\title{
Effect of Iron Supplementation On The Biogas Production and Microbial Community Distribution During Anaerobic Digestion of Food Waste Process
}

Yue Xu

Wuhan Polytechnic University

Rongtang Zhang

Wuhan Polytechnic University

Jiesheng Liu

Wuhan Polytechnic University

Xinghua He

Wuhan Polytechnic University

Haijun Lu

Wuhan Polytechnic University

Na Wei

Wuhan Polytechnic University

Jing Zhang ( $\nabla$ zj220220@126.com )

Wuhan Polytechnic University

\section{Research Article}

Keywords: Food waste, Anaerobic digestion, Iron, Methane, Microbial community

Posted Date: July 6th, 2021

DOI: https://doi.org/10.21203/rs.3.rs-600423/v1

License: (c) (1) This work is licensed under a Creative Commons Attribution 4.0 International License.

Read Full License 


\section{Abstract}

Iron as micronutrients is of great significance for forming a stable and efficient anaerobic digestion of food waste. Aim of this study was to examine the effect of iron supplementation on the mesophilic anaerobic digestion of food waste. Firstly, batch experiments were conducted with different iron concentration at a constant kitchen waste/inoculum ratio $(K / I=1.0)$, which indicated that the effect of iron on anaerobic digestion of was strictly dosage-dependent. Then, anaerobic digestion of food waste was conducted for 50 days in semi-continuous rectors with optimal iron concentration $(2.0 \mathrm{mg} / \mathrm{L})$ under the same conditions. And the semi-continuous rectors obtained a good operation performance with low volatile fatty acids concentration, higher biogas production, high coenzyme $F_{420}$ and dehydrogenase concentrations. Furthermore, two samples taken on 7th day and 50th day were analyzed by highthroughput sequencing, which illustrated that the composition anaerobe community was stable. However, the growth and activity of several syntrophic microbial groups (Aminobacterium, Syntrophomonas, Anaerolineaceae, Methanosaeta, Methanosarcina, Methanobacterium and Methanospirillum), were stimulated by iron supplementation. The shift of microbial community suggested that a high-efficiency microbial community for methane production from food waste was formed by iron supplementation.

\section{Introduction}

The annual generation of food waste (FW) is more than 1.4 billion tons globally, which accounts for approximately $33 \%$ of total annual global food production(Guo et al., 2019; Wong et al., 2018). And what's worse, the FW generation is expected to keep an ever-increasing owing to the rising living standards and population growth(Uçkun Kiran et al., 2014). FW is mainly composed of water, vegetables, fruit, meat, rice, bakery and dairy products, which easily spoil even at room temperature(Uçkun Kiran et al., 2014; Zhang et al., 2020b). Hence, huge FW not only inevitably causes a serious waste of resources, but also causes a series of environmental pollutions(Yue et al., 2020). Now FW management has been an urgent environmental, economic and social problem to be solved around the world(Gaby et al., 2017; Kaur et al., 2019).

Due to rich nutrition, FW is also considered as a typical "misplaced" renewable resource(Bedoić et al., 2020). High water content and high carbohydrate ratio make FW an ideal substrate for anaerobic digestion (AD) to produce methane(Yue et al., 2020; Zhang et al., 2020a). AD not only can realize the harmless and reduction of FW, but also can obtain biogas to realize the resource utilization of FW(Zhang et al., 2020a). Hence, AD has been one of the most suitable and efficient biological treatment for FW(Ren et al., 2018). Nevertheless, $A D$ is a sensitive multi-stage bioprocess including hydrolysis, acidogenesis, acetogenesis and methanogenesis, which depends on different functional microorganism to degrade organic compounds into methane step by step(De Vrieze et al., 2012b). Hence, AD is easily affected by diverse environmental parameters such as $\mathrm{pH}$, temperature, nutrition balance, ammonia, volatile fatty acids(VFAs)(Wang et al., 2018). The microbial population and activity are the core of the digesters to ensure long-term process stability and efficiency(Wang et al., 2018). Nutrition balance as one of environmental parameters is of great importance for the microbial growth during AD process(Choong et 
al., 2016). Besides basic macronutrients such as carbon (C), nitrogen (N), phosphorus (P) and sulphur (S), trace elements (TE) such as nickel (Ni), iron (Fe) and cobalt (Co) play an irreplaceable role in enzymatic synthesis, in respiratory processes, cell structural stability and signal transduction of acidogenesis and methanogenesis(Choong et al., 2016; Eftaxias et al., 2018b; Hijazi et al., 2020; Thanh et al., 2016). In AD process, Fe not only is a component of the majority of metalloenzymes functioning to form the active site, cofactor and the structure itself, but also serves as a redox carrier to transfer electron(Harrop \& Mascharak, 2005; Vintiloiu et al., 2013). $\mathrm{Ni}$ is an important component of coenzyme $\mathrm{F}_{430}$ of methyl reductase which reduces methyl coenzyme $\mathrm{M}$ to methane in all methanogenic pathways(Friedmann et al., 1990; Thanh et al., 2016). It reported that Co is essential composition of corrinoid such as vitamin B12, which can bind to the coenzyme methylase to catalyze methane formation in both acetoclastic methanogens and hydrogenotrophic methanogens(Friedmann et al., 1990). Hence, adequate trace elements could support the microbial metabolism to maintain an effective AD process. However, one of remarkable characteristics of $\mathrm{FW}$ is low trace element, which could rigorously limit its $\mathrm{AD}$ (Hijazi et al., 2020; Zhang \& Loh, 2019). Therefore, supplementation of trace elements is necessary and promising for efficient biogas production of FW.

In the literature, there are a number of reviews on the synergistic effect of trace metals supplementation in $\mathrm{AD}$ (Eftaxias et al., 2018a; Molaey et al., 2018; Schmidt et al., 2018; Wang et al., 2021). To our knowledge, no studies have examined how single iron addition effects the methane production and microbial community during $A D$ of FW. This work reports on the influence of Fe addition on $A D$ performance of FW. The results focus on the effect on biogas quantity, quality, coenzyme $F_{420}$, dehydrogenase concentration and microbial communities, especially methanogens.

\section{Material And Methods}

\subsection{Substrates and inoculum}

Table 1

The characteristics of inoculum sludge and kitchen waste

\begin{tabular}{|lll|}
\hline & Inoculum sludge & Food waste \\
\hline $\mathrm{pH}$ & $7.20 \pm 0.00$ & $6.90 \pm 0.01$ \\
\hline $\mathrm{TS}(\%)$ & $3.86 \pm 0.01$ & $26.84 \pm 0.01$ \\
\hline VS (\%) & $1.44 \pm 0.01$ & $29.36 \pm 0.01$ \\
\hline The moisture content (\%) & $96.14 \pm 0.01$ & $73.16 \pm 0.01$ \\
\hline $\mathrm{C} / \mathrm{N}$ & $7.12 \pm 0.01$ & $20.46 \pm 0.01$ \\
\hline
\end{tabular}

KW was collected from the Wuhan University cafeteria (Wuhan, Hubei province, China). Frist, indigestible substrates such as plastic and chopsticks were removed form FW before being homogenized, then the 
homogenized $\mathrm{KW}$ substrates were kept in a $-20^{\circ} \mathrm{C}$ fridge until required. The microbial inoculum (seed sludge) used was collected from an anaerobic digester in Municipal Engineering Ltd., Wuhan University, Wuhan. The seed sludge was first left undisturbed for $48 \mathrm{~h}$ at $37^{\circ} \mathrm{C}$ to remove endogenous biogas production. Detailed characteristics of the substrate and seed sludge used in this study are presented in Table 1.

\subsection{Batch trials for methane production form $\mathrm{FW}$ in the presence of different concentration of iron}

Batch trials were conducted in a series of $2.0 \mathrm{~L}$ identical serum bottles containing $0.2 \mathrm{~L}$ of seed sludge and $0.8 \mathrm{~L}$ of Milli-Q water to a final working volume of $1.0 \mathrm{~L}$ with $\mathrm{K} / \mathrm{I}$ ratio of 1.0 at $37^{\circ} \mathrm{C}$. Then, different volume of iron supplement solution of $\mathrm{FeCl}_{3} \otimes 6 \mathrm{H}_{2} \mathrm{O}$ in deionized water was added into serum bottles to obtain different concentration of iron $(0,0.5,1.0,2.0,4.0$ and $6.0 \mathrm{mg} / \mathrm{L})$. All experiments were conducted in triplicate. The cumulative biogas production, methane yield, and coenzyme $\mathrm{F}_{420}$ and dehydrogenase were measured after $72 \mathrm{~h}$. No alkalinity or buffering agent was added into the system before the reaction, and the $\mathrm{pH}$ value was not adjusted during the process.

\subsection{Biogas production and microbial community structure in semi-continuous AD experiment with optimal iron concentration}

Semi-continuous rectors were operated to assess the effect of long-term supplementation of iron on methane production and microbial communities during $A D$ of FW. Frist, $0.4 \mathrm{~L}$ of seed sludge and $1.6 \mathrm{~L}$ of Milli-Q water were added in each semi-continuous rector to obtain a $2 \mathrm{~L}$ working volume at $37^{\circ} \mathrm{C}$. Second, each reactor was fed with $\mathrm{K} / \mathrm{I}=1.0$ once a week to maintain a hydraulic retention time of 14 days. Meanwhile, a certain volume of supplement solution of $\mathrm{FeCl}_{3} \triangle 6 \mathrm{H}_{2} \mathrm{O}$ was added into each reactor to the optimal iron concentration obtained by batch trials at 2-day interval. Then reactors were manually shaken for 2 min twice a day. Cumulative biogas production was measured every two days. The methane yield, coenzyme $\mathrm{F}_{420}$ and dehydrogenase concentrations were measured every week. Finally, to characterize the shifts in microbial communities in response to long-term supplementation of iron during AD of FW, samples taken on days 7 and 50 were analyzed via high-throughput sequencing. Three parallel rectors were conducted.

\subsection{Analytical methods}

The total volume of biogas production was measured via the drainage method at each time interval. Biogas samples were collected from the bottle headspaces using aluminum gas. The biogas composition in the biogas samples collected from the bottle headspaces using aluminum gas were quantified by gas chromatography (GC-2030, Tet Instrument, China) equipped with a thermal conductivity detector (TCD) and a TDX-01-21 column $(1 \mathrm{~m} \times 3 \mathrm{~mm})$. The operating temperatures of the injector, column, and detector were kept at 250,200 , and $110^{\circ} \mathrm{C}$, respectively. High purity nitrogen was used as carrier gas at a flow rate of $500 \mathrm{~mL} \cdot \mathrm{min}^{-1}$. 
Volatile fatty acids (VFAs) analysis was performed using a gas chromatography (GC-2030, Tet Instrument, China) equipped with a flame ionization detector (FID). First, the samples were harvested by centrifugation at $14,000 \times \mathrm{g}$ for $15 \mathrm{~min}$, after which the supernatants were filtrated. Then filtrated liquid digestate was mixed with $100 \mathrm{mg} / \mathrm{L}$ of crotonic acid in a volume ratio of 9:1. Finlay, $1 \mu \mathrm{l}$ mixed liquid was injected onto an organic acid separation column $(3 \mathrm{~m} \times 3 \mathrm{~mm})$. The temperatures of the injection port, column, detector was 110,200 and $280^{\circ} \mathrm{C}$, respectively. High purity nitrogen was used as carrier gas (500 $\left.\mathrm{mL} \cdot \mathrm{min}^{-1}\right)$.

Dehydrogenase activity and coenzyme $F_{420}$ were measured via 2,3,5- triphenyltetrazoliumchloride spectrophotometry(Gabbita \& Huang, 1984) and ultraviolet visible light spectrophotometry(Grochowski \& White, 2010), respectively.

\subsection{Metagenomic analysis}

To study the effect of supplementation of iron on microbial communities during AD of FW, sludge samples were taken on days 7 and 50 . The total DNA of each sample was extracted with the TIANamp Bacteria DNA Kit (TIANGEN Biotech Co., Ltd., Beijing, China) following the manufacturers' instructions. The DNA samples were then sent to Shanghai Majorbio Bio-pharm Technology Co., Ltd. (Shanghai, China) for high-throughput sequencing to elucidate the shifts in microbial communities. Microbial 16SrRNA sequences were amplified via polymerase chain reaction (PCR) using the forward ArBa515F (50-GTG CCA GCM GCC GCG GTA A -30) and reverse Arch806R (50- GGA CTA CVS GGG TAT CTA AT-30) primer pair (28). The complete genome of the sampled bacteria was sequenced in an Illumina MiSeq system (PE300, Illumina, San Diego, CA, USA) following the manufacturer's instructions.

\section{Results And Discussion}

\subsection{Batch trials for methane production form FW in the presence of different concentration of iron}

Figure 1 presented biogas production and methane yield with different concentrations of iron. As shown in Fig. $2 \mathrm{~A}$, the biogas production was $1725,1731,1777,1821,1737$ and $1543 \mathrm{ml}$ at $0.0,0.5,1.0,2.0,4.0$ and $6.0 \mathrm{mg} / \mathrm{L}$ of iron, respectively. Although the biogas production increased with the increasing of iron concentration between $0.5 \mathrm{mg} / \mathrm{L}$ to $2.0 \mathrm{mg} / \mathrm{L}$, no significant increase was observed. However, when the iron concentration exceeded $2.0 \mathrm{mg} / \mathrm{L}$, the biogas production was reduced. Figure $1 \mathrm{~B}$ illustrated that low concentrations of iron could enhance methane yield, whereas high concentrations of iron had an inhibitory effect on methane yield. And the maximum methane yield achieved at $2.0 \mathrm{mg} / \mathrm{L}$ of iron was $137.5 \mathrm{ml} / \mathrm{g} \cdot \mathrm{VS}$, which was approximately 1.17 -fold of that in the control. The results are consistent with several previous studies(Choong et al., 2016; Facchin et al., 2013; Meng et al., 2013; Yu et al., 2015). Iron as an indispensable micronutrient could effectively enhance the activity of anaerobes and promote the growth of anaerobes at relatively lower concentration(Choong et al., 2016). However, high concentration of iron is easy to cause a toxicity on anaerobes, inhibit the process of methanogenesis(Choong et al., 
2016; Facchin et al., 2013). Therefore, the effect of iron on KW AD exhibited a hormetic trend with $2.0 \mathrm{mg}$ /L as the threshold.

\subsection{Methane yield and biogas production with optimal iron concentrations in semi-continuous AD experiment}

Figure 2 illustrated the time course of the cumulative biogas production and methane yield profiles with optimal iron concentrations in semi-continuous reactors. First of all, the cumulative biogas production rapidly increased at the beginning, and then gradually stabilized in each cycle. Notably, the total accumulative volume of biogas was about $4100 \mathrm{ml}$ in each cycle, which shown no significant impact on cumulative biogas production in semi-continuous reactors. However, as the reaction progresses, the time needed to reach the maximum biogas accumulation gradually decreased in each cycle. Meanwhile, methane yield gradually increased at the end of each cycle. Methane yield in 7th cycle for iron supplementation group was $168.68 \mathrm{~mL} / \mathrm{g} \cdot \mathrm{VS}$. Compared to the first cycle, methane yield in 7 th cycle increased by $18.6 \%$. These phenomena demonstrated that supplementation of iron in semi-continuous reactors could enhanced methane content of biogas production. Iron could act as cofactors in enzymes that are required during methane fermentation(Yu et al., 2015; Zandvoort et al., 2006). Hence, for FW with low trace element content, supplementation of iron in AD system could enhanced methane yield.

\subsection{Effect of optimal iron concentration on VFAs in semi- continuous AD experiment}

VFAs are the main intermediate products that convert to methane(Tian et al., 2015; Velmurugan et al., 2010). Figure 3 showed the composition and variation of VFAs during AD of KW in semi-continuous AD experiment. As shown in Fig. 3, VFAs were composed of acetic acid, propionic acid and butyric acid. And the acetic acid content is the highest, followed by propionic acid. Besides, the variation of each VFAs was very similar. The concentration of each VFAs in each cycle firstly increased, and then decreased. Notably, as the reaction progresses, the maximum concentration of each VFAs in each cycle decreased. For example, the maximum concentration of acetic acid and propionic acid in last cycle was $0.25 \mathrm{~g} / \mathrm{L}$ and $0.06 \mathrm{mg} / \mathrm{L}$, which was about 0.38 -fold of that in the first cycle, respectively. After 35 days, there was almost no butyric acid. Hence, the iron supplementation promoted VFAs degradation and prevented acid accumulation.

The bidirectional Wood-Ljungdahl (Reductive Acetyl-Coenzyme A) pathway is main route for many acetoclasts and acetogens to convert acetate to $\mathrm{H}_{2}$ and $\mathrm{CO}_{2}$. Enzymes carbon monoxide dehydrogenaseacetyl-coenzyme A synthase (CODH-ACS) and methyl-transferase-Ac Co-A are two important enzymes for this route, which need sufficient iron(Bender et al., 2011; Dobbek et al., 2001). Besides, it reported that reactions yield included into bidirectional Wood-Ljungdahl pathway was very little energy (e.g. acetate to bicarbonate: $+25 \mathrm{~kJ} \Delta \mathrm{G}^{0}$; propionate to acetate: $+18 \mathrm{~kJ} \Delta \mathrm{G}^{0}$ ) (Thauer et al., 1977), which makes the pathway easy to proceed. Therefore, relatively large amounts of iron are expected to be required for growth in acetoclastic/propionoclastic populations. So iron supplementation was beneficial to these enzymes synthesis. 


\subsection{Effect of optimal iron concentration on concentrations coenzyme $\mathrm{F}_{420}$ and dehydrogenase concentrations in semi- continuous AD experiment}

Dehydrogenase is an essential intracellular microbial enzyme(Salazar et al., 2011) and is widely used as an indicator of overall microbial activity occurred intracellularly in all living microbial cells. Moreover, coenzyme $F_{420}$ (7, 8-didemethyl-8-hydroxy-5-deazariboflavin derivative), a special coenzyme for electron transport, only presents in methane-producing microorganisms. Therefore, it is feasible to use coenzyme $F_{420}$ concentration as an indicator of methanogenic activity(Cheng et al., 2007; Whitmore et al., 1986).

Figure 4 shown the time course of coenzyme $\mathrm{F}_{420}$ and dehydrogenase concentration with optimal iron concentration in semi-continuous $A D$ reactors. The variations of coenzyme $F_{420}$ and dehydrogenase concentration were very similar. As the experiment went on, coenzyme $F_{420}$ and dehydrogenase concentration both increased, and obtained their maximum at the end 7th cycle. As shown in Fig. 4, the maximum dehydrogenase concentration was $119.71 \mathrm{mg} / \mathrm{L}$, which increased by $16.3 \%$. The maximum coenzyme $\mathrm{F}_{420}$ concentration in Fe supplementation groups were $0.24 \mu \mathrm{mol} /$. And compared to the first cycle, coenzyme $\mathrm{F}_{420}$ concentration in 7 th cycle increased by $25.68 \%$. As indispensable micronutrient, addition of iron in semi-continuous $A D$ reactors could enhance the activity of anaerobe and promote the growth of anaerobe, especially methanogens(Choong et al., 2016; Eftaxias et al., 2018b; Molaey et al., 2018). These experimental results were consistent with the previous results of cumulative biogas production and methane yield in iron supplementation semi-continuous $A D$ experiment. Increasing of the coenzyme $\mathrm{F}_{420}$ and dehydrogenase concentration indicated a high activity of anaerobe, especially methanogens. Hence, the methane yield increased.

\subsection{Shift of microbial community with optimal iron concentration in semi-continuous AD experiment}

The AD operational conditions could change the microbial community structure, which determine methane production(Town et al., 2014). Two biomass samples collected at days 7 and 50 were analyzed via high-throughput sequencing, after which the resulting gene sequences of individual samples were assigned to different taxa levels (from phyla to genus). In this study, only the major groups (genera and phyla) with a relative abundance of no less than $0.1 \%$ were assigned to said samples. Changes of anaerobe community structure in semi-continuous reactor with optimal iron concentration shown in Fig. 5. As shown in Fig. 5A, the composition anaerobe community at phyla level was stable. The main anaerobe phyla were Synergistetes, Firmicutes, Euryarchaeota, Bacteroidetes and Chloroflexi, which are known to be involved in different phases of the AD process(Nelson et al., 2011). Other phyla such as Proteobacteria, Actinobacteria and Armatimonadetes were identified in minor proportions, which is consistent with previous studies(Ariesyady et al., 2007; Hernon et al., 2006). In the early stage, Synergistetes (ca. 31.02\%) was the most dominant anaerobe at the phylum level, followed by Firmicutes (ca. 23.94\%), Euryarchaeota (ca. 16.44\%), Bacteroidetes (ca. 16.44\%) and Chloroflexi (ca. 4.19\%). 
However, after 50 days, Euryarchaeota grew to be the dominant anaerobe with relative abundances of $32.27 \%$, while the relative abundances of Firmicutes, Synergistetes, Bacteroidetes and Chloroflexi were $22.67 \%, 16.70 \%, 16.25 \%$ and $6.68 \%$, respectively. The result suggested that iron supplementation stimulated Euryarchaeota growth, which was beneficial to enhance methane yield. Conversely, Synergistetes was inhibited by the iron supplementation.

As shown in Fig. 5B, among the Synergistetes phyla, Synergistaceae_uncultured and Aminobacterium were the major genera during the entire process (42). As anaerobic reaction proceeded, the relative abundance of Synergistaceae_uncultured decreased from $25.49-7.5 \%$, which was the main reason for the decreased of the relative abundance of Synergistetes. However, the relative abundance of Aminobacterium increased from 6.29-9.23\%. Meanwhile, the relative abundance of Anaerolineaceae_ (within the phylum of Chloroflexi) and Syntrophomonas (within the phylum of Firmicutes) increased from $3.76 \%$ and $1.43-5.19 \%$ and $7.05 \%$, respectively. Notably, the enhancement of Syntrophomonas growth was strongest. Aminobacterium as a mesophilic amino-acid-degrading bacteria could grow in syntrophic interactions with the hydrogenotroph Methanobacterium by fermentation of a limited range of amino acids(Baena et al., 1998; Chertkov et al., 2010). Protein is an important component of KW; and it could be hydrolyzed to amino acids by hydrolytic bacteria. Hence, the enhancement of Aminobacterium growth stimulated by iron supplementation would guarantee an effectively syntrophic degradation of protein and improve the methane yield from FW. Syntrophomonas, as a syntrophic metabolizer with

hydrogenotrophic methanogens, could convert fatty acids into acetate, $\mathrm{H}_{2}$, formate and $\mathrm{CH}_{4}$ (Zhang et al., 2020c). Therefore, the strongest enhancement of Syntrophomonas growth was beneficial to sustain efficient syntrophic communities of bacteria and archaea to avoid over accumulation of intermediate products such as $\mathrm{H}_{2}$ and VFAs(Zhang et al., 2020c), which was in agreement with the change of VFAs shown in Fig. 4. Besides, it was reported that Anaerolineaceae can use $\mathrm{H}_{2}$ as electron carrier to degrade alkane and long-chain fatty acid (LCFA) into methane by cocultivation with hydrogenotrophic methanogens(Liang et al., 2015; Liang et al., 2016). FW contains a certain amount of lipid, which has high methane yield potential. And lipid initially is hydrolyzed to LCFA by extracellular lipases that are excreted by the acidogenic bacteria and then glycerol, then is converted to acetate and hydrogen $\left(\mathrm{H}_{2}\right)$ through the b-oxidation pathway (syntrophic acetogenesis), and finally to methane $\left(\mathrm{CH}_{4}\right)$ by methanogenic archaea(Alves et al., 2009; Zhang et al., 2020b). Hence, the enhancement of Anaerolineaceae growth would be conducive to the degradation of lipid into methane.

Figure 5B also shown that no significant change of archaeal community structure with iron supplementation in the semi-continuous AD reactors after 50 days. And the phylum Euryarchaeota was primarily represented by the genus Methanosaeta, Methanosarcina Methanobacterium and Methanospirillum. As anaerobic reaction proceeded, the relative abundance of Methanosaeta, Methanosarcina, Methanobacterium and Methanospirillum increased form $15.08 \%, 11.46 \%, 6.34 \%$ and $1.43-25.67 \%, 20.19 \%, 7.73 \%$ and $4.11 \%$, respectively. Obviously, during the AD process, supplementation of iron enhanced these four methanogens growth. And the enhancement of Methanosaeta growth was strongest, followed by Methanosarcina. Notably, Methanosaeta always was the most dominant archaeal 
genus in the semi-continuous AD reactors. Methanosaeta, an acetic-utilization methanogens, could converted acetate into methane, and its importance for methane production(Conklin et al., 2006; De Vrieze et al., 2012a; De Vrieze et al., 2015). This result is consistent with the observations of Zhang et al. (Zhang et al., 2020c). Methanosarcina as second dominant archaeal genus is a type of both acetoclastic and hydrogenotrophic methanogens. It could produce methane from acetate, methanol, monomethylamine, dimethylamine, trimethylamine, $\mathrm{H}_{2} / \mathrm{CO}_{2}$, and $\mathrm{CO}$ (Garcia et al., 2000). Thereby, the enhancement of Methanosaeta and Methanosarcina contributed to avoid over accumulation of VFAs. Besides, Methanospirillum and Methanoculleus detected in this process were both hydrogenotrophic methanogens. But the enchantment of them by iron supplementation was relatively low.

According to the results of the shift of microbial community, iron supplementation affect AD performance by forming a high-efficiency microbial community for methane production from KW. As an essential element for anaerobe, iron can synthesize and activate some key coenzymes involved in methanogenesis, such as such as formylmethanofuran dehydrogenase, hydrogenase, acetyl-coenzyme A synthetase, carbon monoxide dehydrogenase, coenzyme $\mathrm{M}$ methyltransferase, Ferricytochrome $\mathrm{c}, \mathrm{F}_{420} \mathrm{H}_{2}$ dehydrogenase and heterodisulfide reductase (Baek et al., 2019; Qi et al., 2021; Schattauer et al., 2011), which are significant form the acid production to methane formation during AD process. For example, carbon monoxide dehydrogenase plays an important role in methane formation form acetate and acetate production from $\mathrm{H}_{2}, \mathrm{CO}_{2}$ and methanol(Schattauer et al., 2011). Hydrogenase consumes $\mathrm{H}_{2}$ to offer electrons to $\mathrm{CO}_{2}$ to produce methane by hydrogenotrophic methanogens (Choong et al., 2016). In short, the shift of microbial community suggested a dependence of methanogenic performance on iron.

\section{Conclusions}

The effect of iron on $A D$ of KW presented a hormetic pattern. Iron supplementation with concentration of $\leq 2.0 \mathrm{mg} / \mathrm{L}$ enhanced the methane yield, whereas higher iron concentration weakened methane yield. Digesters with optimal iron concentration maintained stable and efficient AD performance presented by high methane yield, low VFAs concentrations, high coenzyme $F_{420}$ concentrations and high dehydrogenase concentrations. Pyrosequencing results revealed that the phylum Synergistetes was the most dominant at the beginning, whereas Euryarchaeota became dominant phyla after 50 days. Iron supplementation improve the activity of bacteria (Aminobacterium, Syntrophomonas and Anaerolineaceae) and archaea (Methanosaeta, Methanosarcina Methanobacterium and Methanospirillum), which formed a high-efficiency microbial community for methane production from KW by AD.

\section{Declarations}

Authors' contributions Yue Xu and Jing Zhang: Conceptualization, Methodology, Validation, Formal analysis, Investigation, Data curation, Writing - original draft, Writing - review \& editing; Rongtang Zhang, 
Liujie Sheng, Xinghua He, Haijun Lu and Na Wei: Writing - review \& editing, Supervision. All authors read and approved the final manuscript.

Funding This work was supported by Research and Innovation Initiatives of WHPU and Department of Housing and Urban-Rural Development of Hubei Province.

Data availability The datasets used and/or analyzed during the current study are available from the corresponding author on reasonable request.

\section{Compliance with ethical standards}

Ethics approval and consent to participate Not applicable.

Consent for publication Not applicable.

Competing interests The authors declare that they have no competing interests.

\section{References}

1. Alves MM, Pereira MA, Sousa DZ, Cavaleiro AJ, Picavet M, Smidt H, Stams AJM (2009) Waste lipids to energy: how to optimize methane production from long-chain fatty acids (LCFA). Microb Biotechnol 2(5):538-550

2. Ariesyady HD, Ito T, Okabe S (2007) Functional bacterial and archaeal community structures of major trophic groups in a full-scale anaerobic sludge digester. Water Res 41(7):1554-1568

3. Baek G, Kim J, Lee C (2019) A review of the effects of iron compounds on methanogenesis in anaerobic environments. Renew Sust Energ Rev 113:109282

4. Baena S, Fardeau ML, Labat M, Ollivier B, Thomas P, Garcia JL, Patel BK (1998) Aminobacterium colombiensegen. nov. sp. nov., an amino acid-degrading anaerobe isolated from anaerobic sludge. Anaerobe 4(5):241-250

5. Bedoić R, Špehar A, Puljko J, Čuček L, Ćosić B, Pukšec T, Duić N (2020) Opportunities and challenges: Experimental and kinetic analysis of anaerobic co-digestion of food waste and rendering industry streams for biogas production. Renew Sust Energ Rev 130:109951

6. Bender G, Pierce E, Hill JA, Darty JE, Ragsdale SW (2011) Metal centers in the anaerobic microbial metabolism of $\mathrm{CO}$ and $\mathrm{CO} 2$. Metallomics: integrated biometal science 3(8):797-815

7. Cheng Y-h, Sang S-x, Huang H-z, Liu X-j, Ouyang J-b (2007) Variation of Coenzyme F420 Activity and Methane Yield in Landfill Simulation of Organic Waste. J China Univ Min Tech 17(3):403-408

8. Chertkov O, Sikorski J, Brambilla E, Lapidus A, Copeland A, Del Rio G, Nolan T, Lucas M, Tice S, Cheng H, Han J-F, Detter C, Bruce JC, Tapia D, Goodwin R, Pitluck L, Liolios S, Ivanova K, Mavromatis N, Ovchinnikova K, Pati G, Chen A, Palaniappan A, Land K, Hauser M, Chang L, Jeffries Y-J, Spring CD, Rohde S, Göker M, Bristow M, Eisen J, Markowitz JA, Hugenholtz V, Kyrpides P, Klenk NC, H.-P (2010) 
Complete genome sequence of Aminobacterium colombiense type strain (ALA-1T). Stand Genomic Sci 2(3):280-289

9. Choong YY, Norli I, Abdullah AZ, Yhaya MF (2016) Impacts of trace element supplementation on the performance of anaerobic digestion process: A critical review. Bioresour Technol 209:369-379

10. Conklin A, Stensel HD, Ferguson J (2006) Growth kinetics and competition between Methanosarcina and Methanosaeta in mesophilic anaerobic digestion. Water Environ Res 78(5):486-496

11. De Vrieze J, Hennebel T, Boon N, Verstraete W (2012a) Methanosarcina: the rediscovered methanogen for heavy duty biomethanation. Bioresour Technol 112:1-9

12. De Vrieze J, Hennebel T, Boon N, Verstraete W (2012b) Methanosarcina: The rediscovered methanogen for heavy duty biomethanation. Biores Technol 112:1-9

13. De Vrieze J, Saunders AM, He Y, Fang J, Nielsen PH, Verstraete W, Boon N (2015) Ammonia and temperature determine potential clustering in the anaerobic digestion microbiome. Water Res 75:312-323

14. Dobbek H, Svetlitchnyi V, Gremer L, Huber R, Meyer O (2001) Crystal structure of a carbon monoxide dehydrogenase reveals a [Ni-4Fe-5S] cluster. Science 293(5533):1281-1285

15. Eftaxias A, Diamantis V, Aivasidis A (2018a) Anaerobic digestion of thermal pre-treated emulsified slaughterhouse wastes (TESW): Effect of trace element limitation on process efficiency and sludge metabolic properties. Waste Manage 76:357-363

16. Eftaxias A, Diamantis V, Aivasidis A (2018b) Anaerobic digestion of thermal pre-treated emulsified slaughterhouse wastes (TESW): Effect of trace element limitation on process efficiency and sludge metabolic properties. Waste Manage 76:357-363

17. Facchin V, Cavinato C, Fatone F, Pavan P, Cecchi F, Bolzonella D (2013) Effect of trace element supplementation on the mesophilic anaerobic digestion of foodwaste in batch trials: The influence of inoculum origin. Biochem Eng J 70:71-77

18. Friedmann HC, Albrecht K, Thauer RK (1990) Structure and function of the nickel porphinoid, coenzyme F430, and of its enzyme, methyl coenzyme M reductase. Fems Microbiol Rev (3-4), 3-4

19. Gabbita KV, Huang JYC (1984) A Modified Method for Determination of Dehydrogenases Activity of Activated-Sludge. Toxicol Environ Chem 8(2-3):119-132

20. Gaby JC, Zamanzadeh M, Horn SJ (2017) The effect of temperature and retention time on methane production and microbial community composition in staged anaerobic digesters fed with food waste. Biotechnology for biofuels 10:302

21. Garcia JL, Patel BK, Ollivier B (2000) Taxonomic, phylogenetic, and ecological diversity of methanogenic Archaea. Anaerobe 6(4):205-226

22. Grochowski LL, White RH (2010) Biosynthesis of the Methanogenic Coenzymes. Comprehensive Natural Products li: Chemistry and Biology, Vol 7: Cofactors, 711-748

23. Guo H, Zhao Y, Damgaard A, Wang Q, Lu W, Wang H, Christensen TH (2019) Material flow analysis of alternative biorefinery systems for managing Chinese food waste. Resour Conserv Recy 149:197- 
24. Harrop TC, Mascharak PK (2005) Structural and spectroscopic models of the A-cluster of acetyl coenzyme a synthase/carbon monoxide dehydrogenase: Nature's Monsanto acetic acid catalyst. Coord Chem Rev 249(24):3007-3024

25. Hernon F, Forbes C, Colleran E (2006) Identification of mesophilic and thermophilic fermentative species in anaerobic granular sludge. Water science technology: a journal of the International Association on Water Pollution Research 54(2):19-24

26. Hijazi O, Abdelsalam E, Samer M, Amer BMA, Yacoub IH, Moselhy MA, Attia YA, Bernhardt H (2020) Environmental impacts concerning the addition of trace metals in the process of biogas production from anaerobic digestion of slurry. J Clean Prod 243:118593

27. Kaur G, Luo L, Chen G, Wong JWC (2019) Integrated food waste and sewage treatment - A better approach than conventional food waste-sludge co-digestion for higher energy recovery via anaerobic digestion. Bioresour Technol 289:121698

28. Liang B, Wang LY, Mbadinga SM, Liu JF, Yang SZ, Gu JD, Mu BZ (2015) Anaerolineaceae and Methanosaeta turned to be the dominant microorganisms in alkanes-dependent methanogenic culture after long-term of incubation. AMB Express 5(1):117

29. Liang B, Wang LY, Zhou Z, Mbadinga SM, Zhou L, Liu JF, Yang SZ, Gu JD, Mu BZ (2016) High Frequency of Thermodesulfovibrio spp. and Anaerolineaceae in Association with Methanoculleus spp. in a Long-Term Incubation of n-Alkanes-Degrading Methanogenic Enrichment Culture. Frontiers in microbiology 7:1431

30. Meng X, Zhang Y, Li Q, Quan X (2013) Adding Fe0 powder to enhance the anaerobic conversion of propionate to acetate. Biochem Eng J 73:80-85

31. Molaey R, Bayrakdar A, Sürmeli R, Çalli B (2018) Influence of trace element supplementation on anaerobic digestion of chicken manure: Linking process stability to methanogenic population dynamics. J Clean Prod 181:794-800

32. Nelson MC, Morrison M, Yu Z (2011) A meta-analysis of the microbial diversity observed in anaerobic digesters. Bioresource Technol 102(4):3730-3739

33. Qi Q, Sun C, Zhang J, He Y, Tong W, Y (2021) Internal enhancement mechanism of biochar with graphene structure in anaerobic digestion: The bioavailability of trace elements and potential direct interspecies electron transfer. Chem Eng J 406:126833

34. Ren Y, Yu M, Wu C, Wang Q, Gao M, Huang Q, Liu Y (2018) A comprehensive review on food waste anaerobic digestion: Research updates and tendencies. Bioresour Technol 247:1069-1076

35. Salazar S, Sanchez LE, Alvarez J, Valverde A, Galindo P, Igual JM, Peix A, Santa-Regina I (2011) Correlation among soil enzyme activities under different forest system management practices. Ecol Eng 37(8):1123-1131

36. Schattauer A, Abdoun E, Weiland P, Plöchl M, Heiermann M (2011) Abundance of trace elements in demonstration biogas plants. Biosyst Eng 108(1):57-65 
37. Schmidt T, McCabe BK, Harris PW, Lee S (2018) Effect of trace element addition and increasing organic loading rates on the anaerobic digestion of cattle slaughterhouse wastewater. Bioresour Technol 264:51-57

38. Thanh PM, Ketheesan B, Yan Z, Stuckey D (2016) Trace metal speciation and bioavailability in anaerobic digestion: A review. Biotechnol Adv 34(2):122-136

39. Thauer RK, Jungermann K, Decker K (1977) Energy conservation in chemotrophic anaerobic bacteria. Bacteriol Rev 41(1):100-180

40. Tian H, Duan N, Lin C, Li X, Zhong M (2015) Anaerobic co-digestion of kitchen waste and pig manure with different mixing ratios. J Biosci Bioeng 120(1):51-57

41. Town JR, Links MG, Fonstad TA, Dumonceaux TJ (2014) Molecular characterization of anaerobic digester microbial communities identifies microorganisms that correlate to reactor performance. Bioresource Technol 151:249-257

42. Uçkun Kiran E, Trzcinski AP, Ng WJ, Liu Y (2014) Bioconversion of food waste to energy: A review. Fuel 134:389-399

43. Velmurugan B, Arathy EC, Hemalatha R, Philip JE, Ramanujam RA (2010) Anaerobic co-digestion of fruit and vegetable wastes and primary sewage sludge. J Environ Eng Sci 52(1):19-22

44. Vintiloiu A, Boxriker M, Lemmer, Oechsner H, Jungbluth T, Mathies (2013) Effect of ethylenediaminetetraacetic acid (EDTA) on the bioavailability of trace elements during anaerobic digestion. Chem Eng J

45. Wang P, Wang H, Qiu Y, Ren L, Jiang B (2018) Microbial characteristics in anaerobic digestion process of food waste for methane production-A review. Bioresour Technol 248:29-36

46. Wang S, Wang J, Li J, Hou Y, Shi L, Lian C, Shen Z, Chen Y (2021) Evaluation of biogas production potential of trace element-contaminated plants via anaerobic digestion. Ecotox Environ Safe 208:111598

47. Whitmore TN, Etheridge SP, Stafford DA, Leroff UEA, Hughes D (1986) The evaluation of anaerobic digester performance by coenzyme F420 analysis. Biomass 9(1):29-35

48. Wong JWC, Kaur G, Mehariya S, Karthikeyan OP, Chen G (2018) Food waste treatment by anaerobic co-digestion with saline sludge and its implications for energy recovery in Hong Kong. Bioresour Technol 268:824-828

49. Yu B, Shan A, Zhang D, Lou Z, Yuan H, Huang X, Zhu N, Hu X (2015) Dosing time of ferric chloride to disinhibit the excessive volatile fatty acids in sludge thermophilic anaerobic digestion system. Bioresour Technol 189:154-161

50. Yue L, Cheng J, Zhang H, Yuan L, Hua J, Dong H, Li Y-y, Zhou J (2020) Inhibition of NVanillylnonanamide in anaerobic digestion of lipids in food waste: Microorganisms damage and blocked electron transfer. J Hazard Mater 399:123098

51. Zandvoort MH, van Hullebusch ED, Fermoso FG, Lens PNL (2006) Trace Metals in Anaerobic Granular Sludge Reactors: Bioavailability and Dosing Strategies. Eng Life Sci 6(3):293-301 
52. Zhang J, Zhang R, He Q, Ji B, Wang H, Yang K (2020a) Adaptation to salinity: Response of biogas production and microbial communities in anaerobic digestion of kitchen waste to salinity stress. $J$ Biosci Bioeng 130(2):173-178

53. Zhang J, Zhang R, Wang H, Yang K (2020b) Direct interspecies electron transfer stimulated by granular activated carbon enhances anaerobic methanation efficiency from typical kitchen waste lipid-rapeseed oil. Sci Total Environ 704:135282

54. Zhang L, Loh K-C (2019) Synergistic effect of activated carbon and encapsulated trace element additive on methane production from anaerobic digestion of food wastes - Enhanced operation stability and balanced trace nutrition. Biores Technol 278:108-115

55. Zhang W, Li L, Wang X, Xing W, Li R, Yang T, Lv D (2020c) Role of trace elements in anaerobic digestion of food waste: Process stability, recovery from volatile fatty acid inhibition and microbial community dynamics. Bioresour Technol 315:123796

\section{Figures}

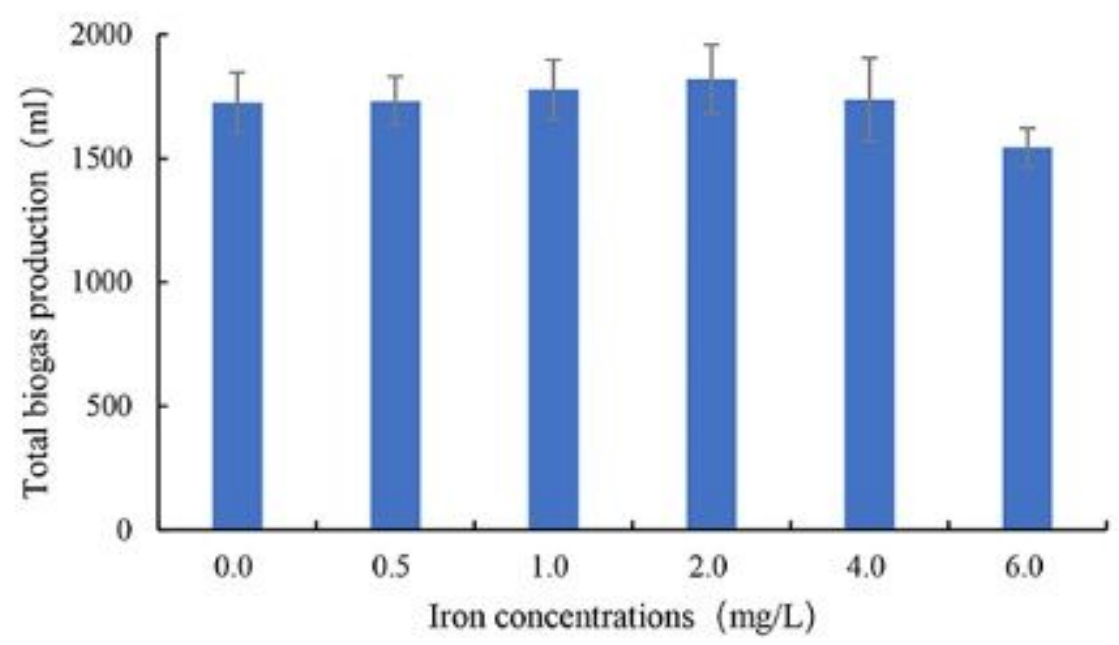

A

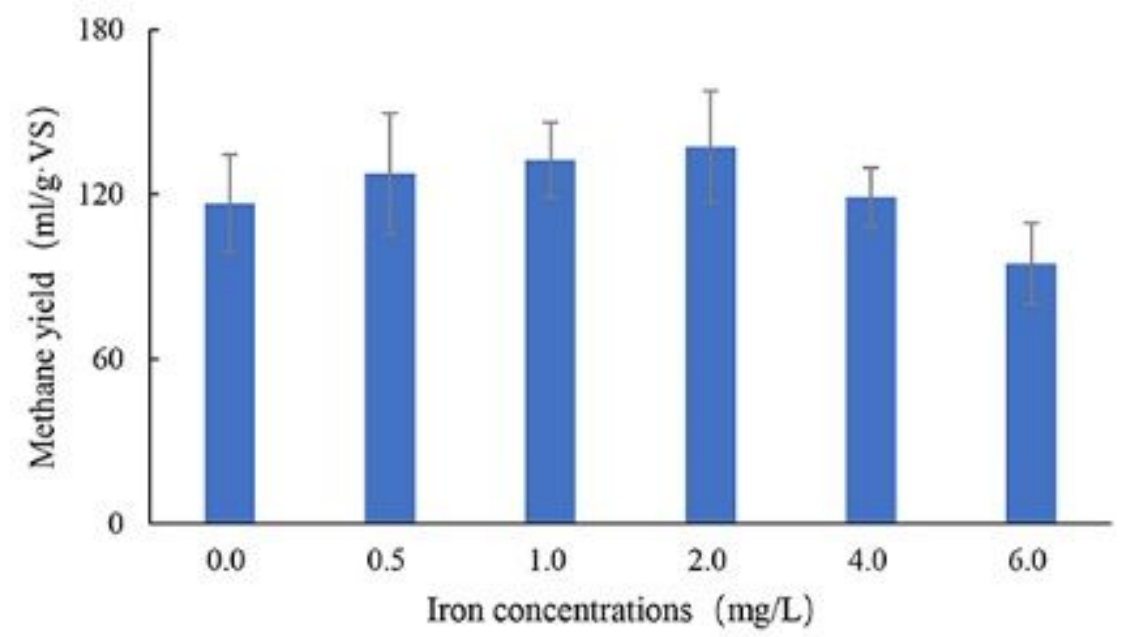

B 
Figure 1

Total biogas production (A) and methane yield (B) under different Iron concentrations

- Cumulative biogas production $=$ Methane yield

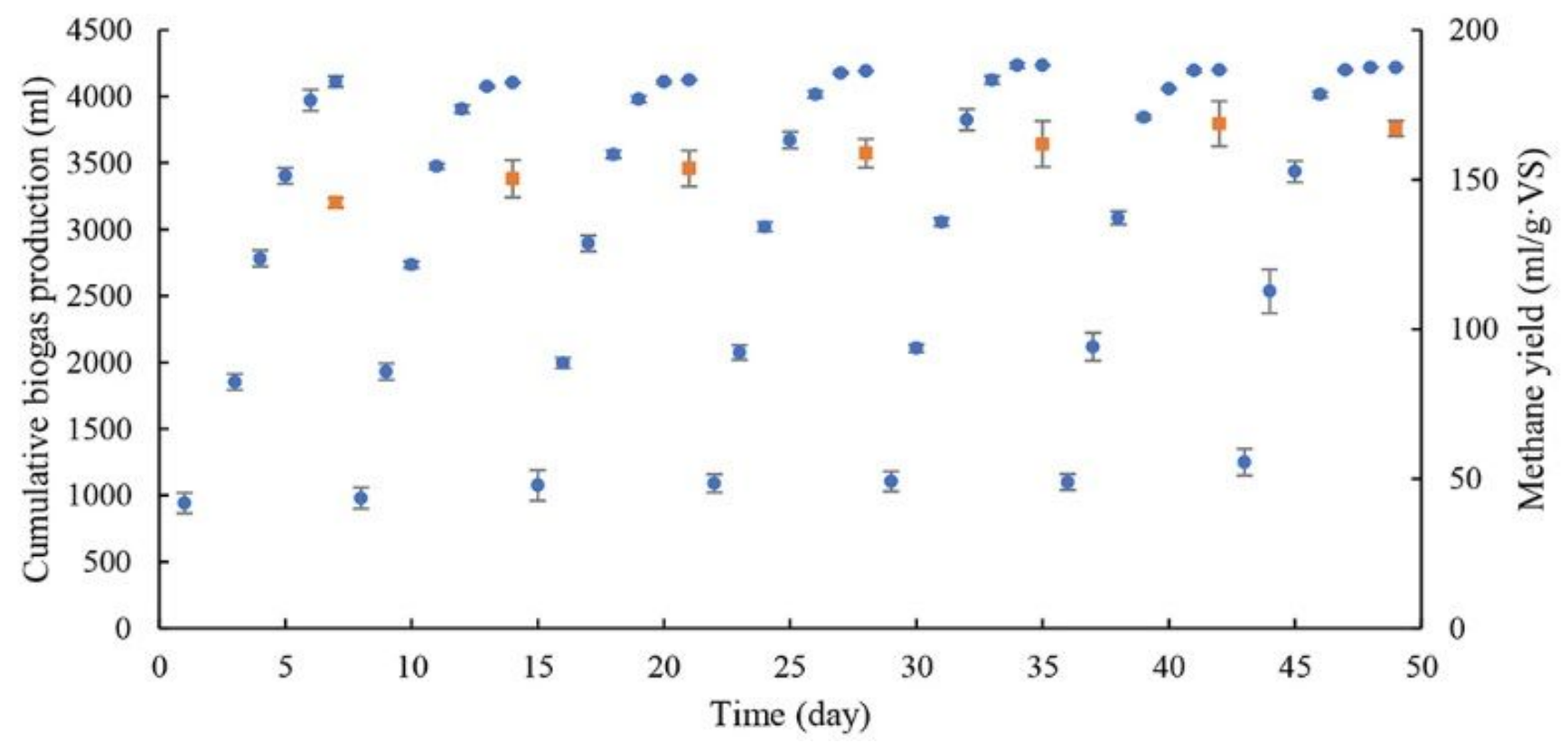

Figure 2

Changes in cumulative biogas production and methane yield profiles with optimal iron concentrations in semi-continuous reactor 


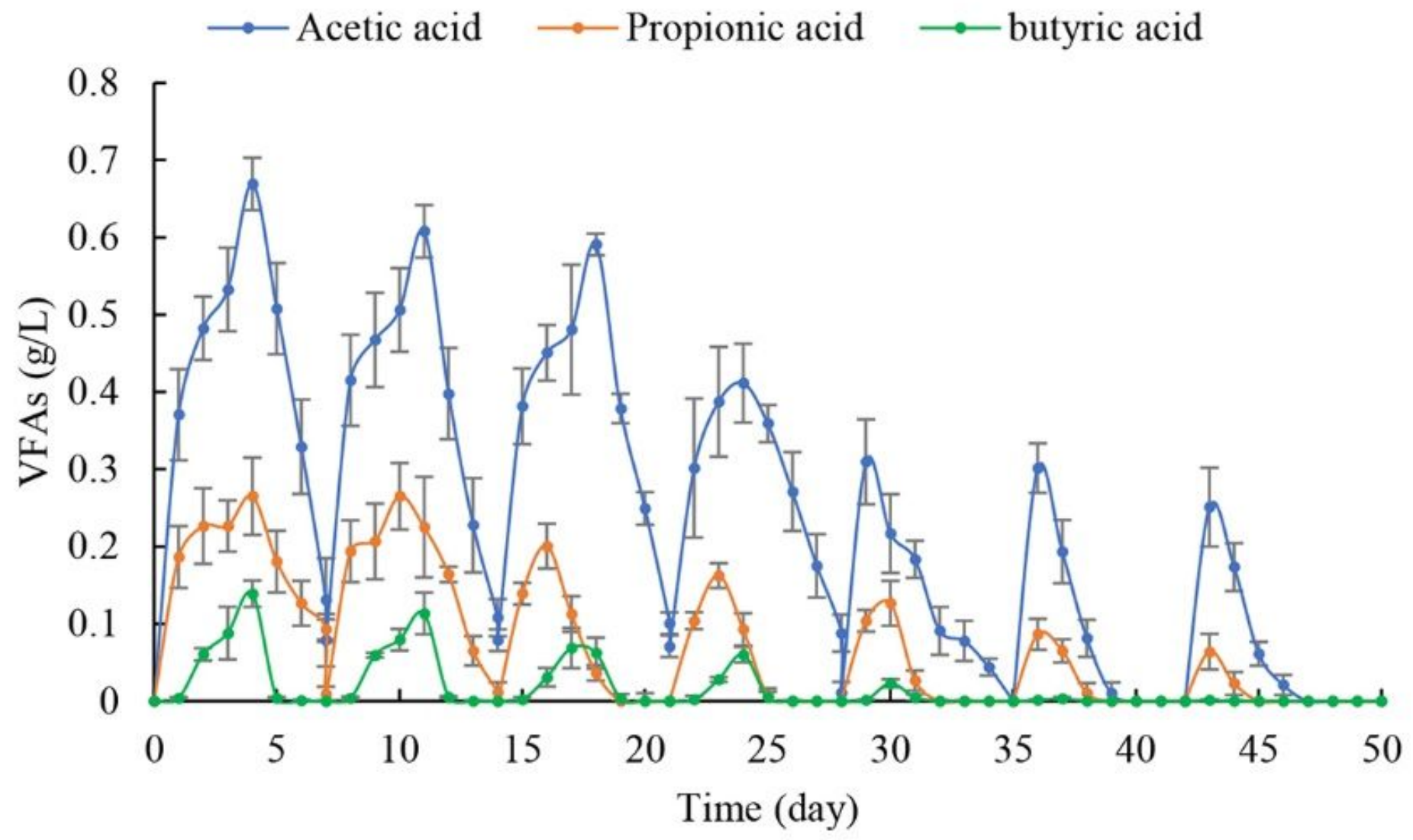

Figure 3

The composition and variation of VFAs during AD of KW in semi-continuous AD experiment 


\section{- Coenzyme F420 • Dehydrogenase}

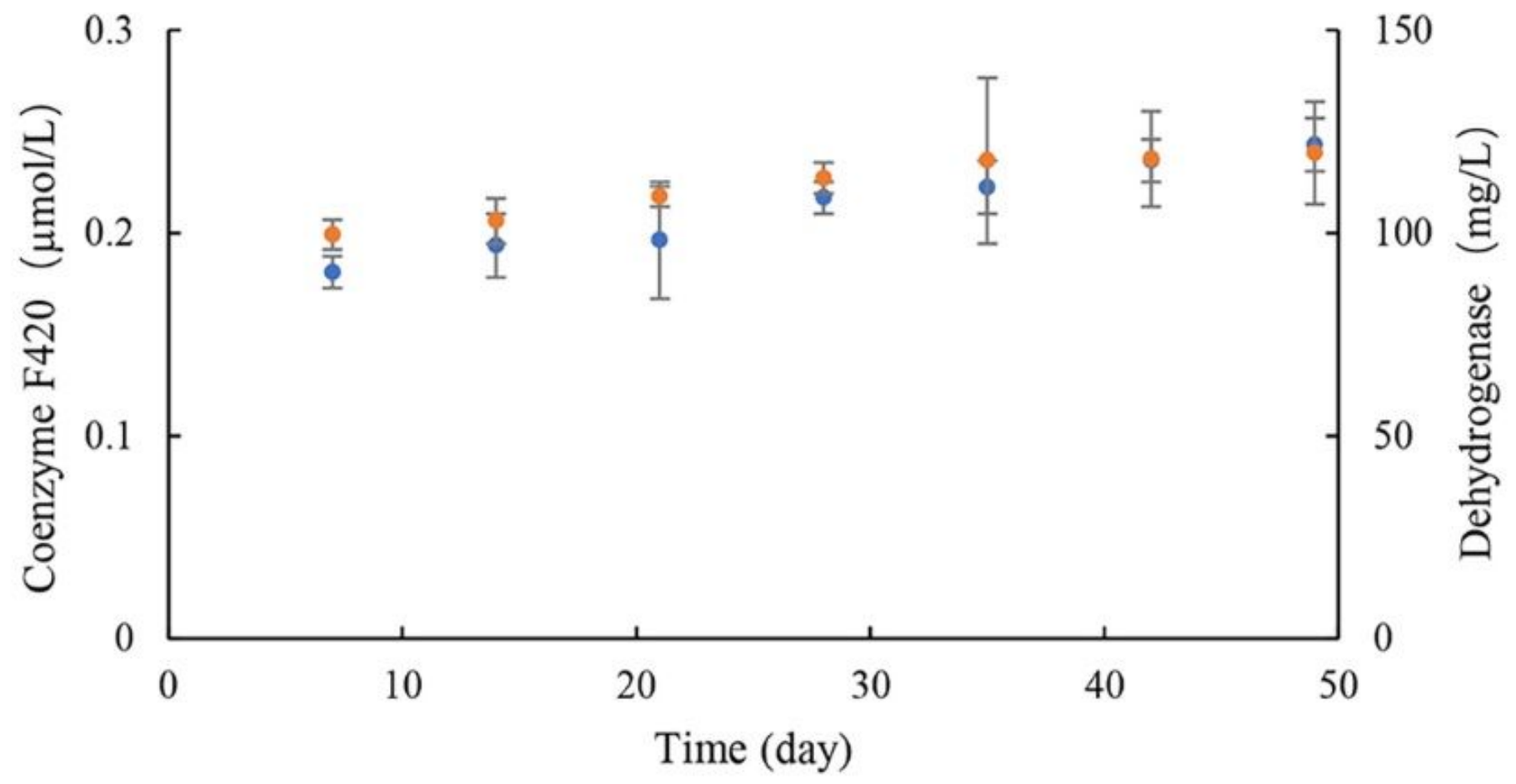

Figure 4

The variation of Coenzyme F420 and dehydrogenase concentrations during AD of KW in semi-continuous $A D$ experiment 


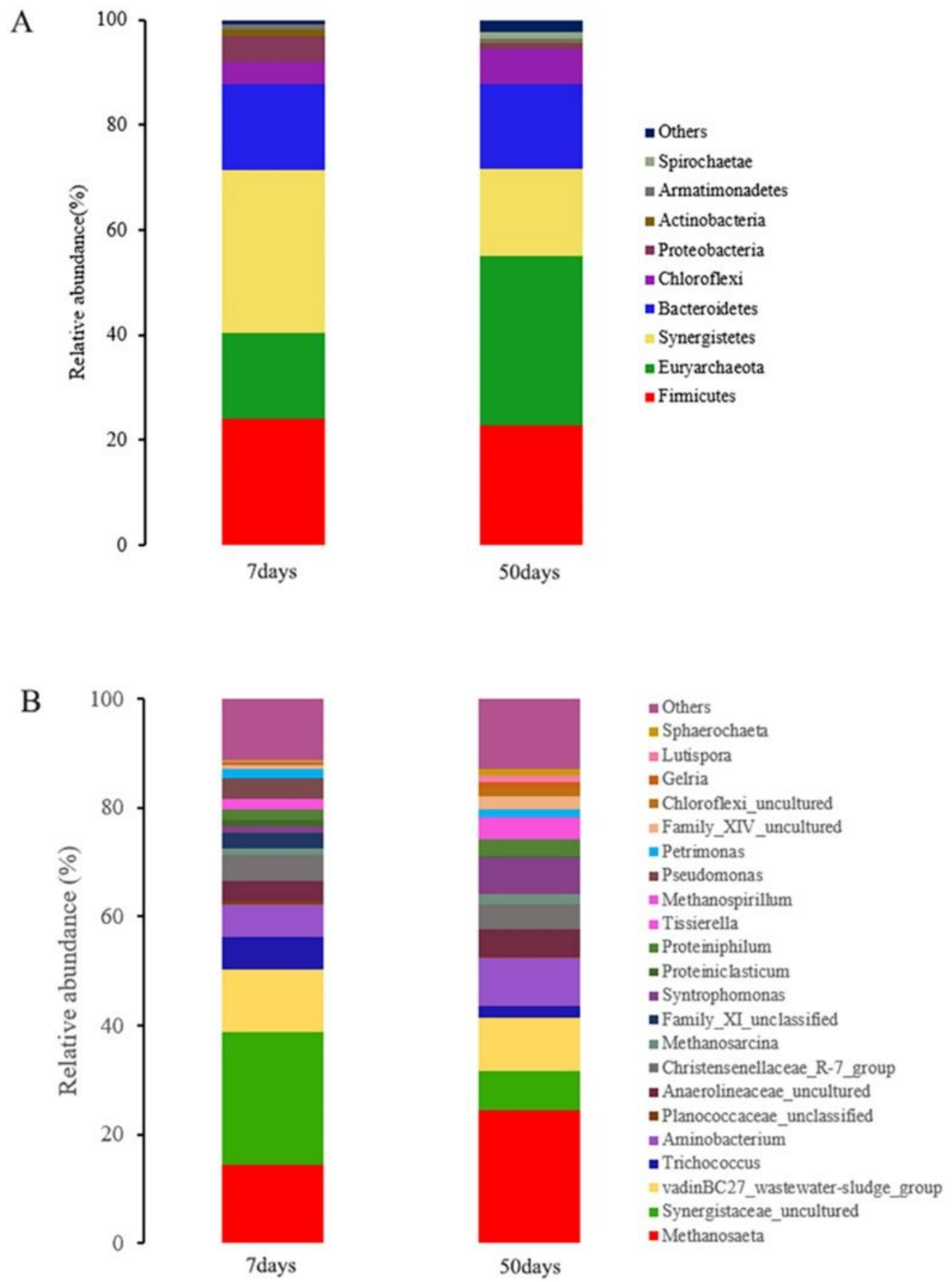

Figure 5

The shift of microbial community at phylum-level (A) and genus-level (B) 